\title{
Osseous Endometrial Metaplasia in a Case of Primary Infertility
}

\author{
Arzoo M Chadha ${ }^{1}$, Mehul Salve ${ }^{2}$, Deepti Shrivastava ${ }^{3}$, Asawari A Deo ${ }^{4}$
}

\begin{abstract}
Endometrial osseous metaplasia is a rare pathological condition characterized by the presence of bone-like tissue in the endometrium and can be a cause for menstrual abnormalities and infertility as bone in the endometrium can act like an intrauterine contraceptive device. It is a rare entity with an estimated incidence of 3/10,000. Exact etiopathogenesis is not known but the most accepted theory is metaplasia of stromal cells into osteoblast cells, which results in the formation of bones. The possibility of malignant mixed Mullerian tumor should also be kept in mind. Here, we report one such case of primary infertility in a 27-year-old woman presenting with history of oligomenorrhea. Endometrial stromal (osseous) metaplasia is a condition that is rarely seen and may have a potential to be overlooked and misdiagnosed. It is important to recognize the non-neoplastic nature of this condition to avoid misinterpretation as malignant mixed Mullerian tumor of the uterus. Its contraceptive nature makes it an extremely rare but treatable cause of infertility. Complete removal of bony spicules by hysteroscopy can help regain fertility. Keywords: Endometrium, Infertility, Metaplasia, Osseous.

International Journal of Infertility and Fetal Medicine (2019): 10.5005/jp-journals-10016-1189
\end{abstract}

\section{INTRODUCTION}

Endometrial osseous metaplasia is a rare pathological disorder characterized by the presence of the bone-like tissue in the endometrium and may cause menstrual irregularities and infertility, because bone in the endometrium may serve as an intrauterine contraceptive tool. ${ }^{1}$ It is a rare entity with an estimated incidence of $3 / 10,000 .^{2}$

Exact etiopathogenesis is not established but the most widely recognized hypothesis is metaplasia of stromal cells into osteoblast cells leading to bone formation. We should always bear in mind the risk of malignant mixed Mullerian tumor. ${ }^{3}$

Here, in a 27-year-old woman with history of oligomenorrhea, we report one such case of primary infertility.

\section{Case Description}

A 27-year-old patient presented to gynecology outpatient department with the history of infertility. She was married for 8 years. She gave history of no previous conception. She underwent dilatation and curettage ( $D$ and $C$ ) 6 months ago. The $D$ and $C$ showed no endometrium. Post $D$ and $C$ she complained of scanty flow during menstruation lasting only for 1 day every 28-30 days.

Her general and bimanual assessments were regular.

Her hematological parameters in practice were normal, and no anomalies were found.

The patient did not have any warning symptoms or laboratory results of calcium deficiency.

Evaluation by ultrasonography showed evidence of calcified foci $2.2 \mathrm{~cm} \times 1.8 \mathrm{~cm}$ in the endometrium. The uterus was anteverted, anteflexed, and normal in size.

The patient was subjected to diagnostic hysteroscopy.

Hysteroscopy was done using rigid hysteroscope (Karl Storz) with saline as distending media. The endometrial cavity revealed few small, hard bony spicules, which were removed and submitted for histopathological study and cartridge-based nucleic acid
${ }^{1-3}$ Department of Obstetrics and Gynaecology, Jawaharlal Nehru Medical College, Sawangi, Wardha, Maharashtra, India

${ }^{4}$ Department of Obstetrics and Gynaecology, Datta Meghe Institute of Medical Sciences, Nanded, Wardha, Maharashtra, India

Corresponding Author: Arzoo M Chadha, Department of Obstetrics and Gynaecology, Jawaharlal Nehru Medical College, Sawangi, Wardha, Maharashtra, India, Phone: +918076128624, e-mail: drarzoochadha@ gmail.com

How to cite this article: Chadha AM, Salve M, Shrivastava D, et al. Osseous Endometrial Metaplasia in a Case of Primary Infertility. Int J Infertil Fetal Med 2019;10(3):52-53.

Source of support: Nil

Conflict of interest: None

amplification test (CBNAAT) testing. Endocervix and uterine cavity showed multiple adhesions. B/L ostia were not visualized.

On the basis of the imaging findings and clinical findings, the presumptive diagnosis of endometrial osseous metaplasia was made.

On histopathology, no endometrium was seen. No inflammatory reaction, products of conception, or signs of necrosis were seen. No evidence of granuloma was seen.

CBNAAT was negative for Mycobacterium tuberculosis complex (MTB) (Fig. 1)

\section{Discussion}

Endometrium osseous metaplasia is defined as an endogenous non-neoplastic pathological disorder, as there is no tissue reaction in the endometrial tissue. ${ }^{1}$

Endometrial ossification is a unusual clinical phenomenon. It is also identified with various other names such as endometrial ossification, intrauterine ectopic bone, and intrauterine heterotopic bone. Ossification can also be observed at other locations including the cervix, ovary, and vagina. ${ }^{2}$ 

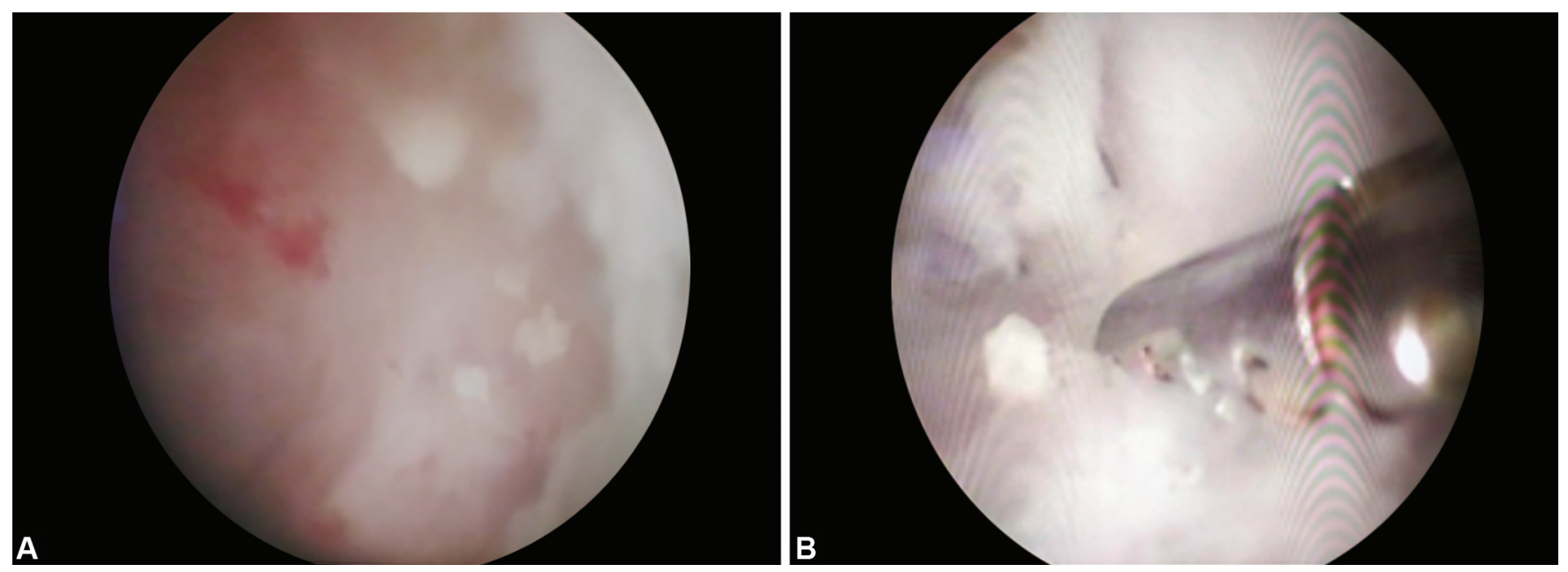

Figs $1 \mathrm{~A}$ and B: Hysteroscopic images of uterine wall calcification s/o endometrial osseous metaplasia

Majority of the affected patients are in the reproductive age group, though a rare case of postmenopausal osseous metaplasia of the endometrium in a 62-year-old woman who had a history of abortion 37 years ago also has been reported by Shimizu et al. ${ }^{2,4}$

In majority of the reported cases, the osseous changes in the endometrium were followed by a previous history of abortion. Majority of the patients are in the reproductive age group with history of first trimester abortion either therapeutic or spontaneous. The time period between a previous abortion and the occurrence of endometrial ossification in the reproductive age group ranges from 8 weeks to 15 years. ${ }^{3}$

Infertility is the principal result of the presence of bone tissue in the uterine cavity. Due to the correlation between bone tissue activity and its intrauterine contraceptive system (IUCD)-like effect, the relationship of osseous metaplasia and infertility appears. ${ }^{5}$

Common clinical presentations are infertility (most common) followed by menstrual irregularities, pelvic pain, dyspareunia, and vaginal discharge. ${ }^{6}$ Our case presented with primary infertility and oligomenorrhea.

Bhatia and Hoshiko incidentally discovered endometrial and cervical osseous metaplasia in a 24-year-old woman. ${ }^{6}$

Cayuela et al. studied the DNA pattern in a 27-year-old woman who was diagnosed to have endometrial osseous metaplasia following first trimester abortion and found the same DNA pattern in the blood of the patient and in the endometrial biopsy including the bone removed from the endometrium. ${ }^{7}$

Heterotopia, dystrophic calcifications, and postabortive endometritis, metastatic calcification, healing tissue metaplasia, prolonged postabortion hormone therapy, and retained fetal bone are the common hypotheses suggested for this disorder. Endometrial tuberculosis should be excluded, as it can lead to infertility, calcification, and subsequent ossification, especially in India. ${ }^{6}$

Chronic endometritis also induces the proliferation of mesenchymal cells that have intrinsic metaplasia properties and can differentiate between either chondroblasts or osteoblasts. ${ }^{3}$

Certain potential diagnoses include: involvement of foreign bodies, Asherman's disease, calcified submucosal fibrosis, and tumors of the Müllerian origin. ${ }^{3,8}$
The treatment for this disorder should be accomplished by hysteroscopic removal of osseous fragments to be submitted for histopathological examination, or by uterine curettage as a second choice. ${ }^{2,3}$ In the present case, the first alternative was adopted.

\section{Conclusion}

Endometrial stromal (osseous) metaplasia is a widely ignored and misdiagnosed disease. Recognition of the non-neoplastic existence of this condition is necessary to prevent misinterpretation as a malignant mixed Mullerian tumor in the uterus. Its contraceptive nature makes it an exceedingly rare but treatable cause of infertility. Total hysteroscopic removal of bony spicules may help to restore fertility.

\section{References}

1. Patil S, Narchal S, Paricharak D, et al. Endometrial osseous metaplasia: case report with literature review. Ann Med Health Sci Res 2013;3(Suppl 1):S10-S12. DOI: 10.4103/2141-9248.121209.

2. Shah NH, Kale KG, Shah VN. Osseous metaplasia of endometrium: a rare cause of secondary infertility. Int J Reprod Contracept Obstet Gynecol 2016;5(2):532-535. DOI: 10.18203/2320-1770. ijrcog20160405.

3. Umashankar T, Patted S, Handigund RS. Endometrial osseous metaplasia: clinicopathological study of a case and literature review. J Hum Reprod Sci 2010;3(2):102. DOI: 10.4103/0974-1208.69329.

4. Shimizu M,Nakayama M.Endometrial ossification in a postmenopausal woman. J Clin Pathol 1997;50(2):171-172. DOI: 10.1136/jcp.50.2.171.

5. Nicolae A, Preda O, Nogales FF. Endometrial metaplasias and reactive changes: a spectrum of altered differentiation. J Clin Pathol 2011;64(2):97-106. DOI: 10.1136/jcp.2010.085555. Available from: https://jcp.bmj.com/content/64/2/97.long.

6. Bhatia NN, Hoshiko MG. Uterine osseous metaplasia. Obstet Gynecol 1982;60(2):256-259.

7. Cayuela E, Perez-Medina T, Vilanova J, et al. True osseous metaplasia of the endometrium: the bone is not from a fetus. Fertil Steril 2009;91(4):1293.e1-1293.e4. DOI: 10.1016/j.fertnstert.2008.12.026.

8. Shroff CP, Kudterkar NG, Badhwar VR. Endometrial ossificationreport of three cases with literature review. Indian J Pathol Microbiol 1985;28(1):71-74. 